\title{
ANALYTICAL MODEL OF TEMPERATURE DISTRIBUTION OVER AN ELECTRONIC CIRCUIT BOARD
}

\begin{abstract}
V. V. Vlassov
INPE - Instituto Nacional de Pesquisas Espaciais ETE - Coordenadoria de Engenharia e

Tecnologia Espacial

DMC - Divisão de Controle e Mecânica Orbital

Av. Dos Astronautas, 1758

12227-010, São José dos Campos, SP, Brasil

vlassov@dem.inpe.br

ABSTRACT

The thermal model of an electronic circuit board with installed heat dissipating components is presented as a two-dimension steady-state heat conduction problem with multiple sources distributed over a rectangular region. The corresponding energy equation includes a source term and a temperature-dependent term to account for linear heat transfer in z-direction. Boundary conditions are of first type with unique temperature along the perimeter. The integral-transform technique is applied to obtain closed-form integral solution. Assuming that all dissipated components have a rectangular contact area with the plate, multiple integrals for each dissipated subregion are easily found. A temperature map over the board is calculated from the closed expression with a triple sum of series with respect to each coordinate and source. The error is evaluated by the estimation of the truncated terms. The solution was applied to obtain the temperature distribution over the electronic Driver Plate of the CEP block of the CIMEX Brazilian experiment for flight qualifying of the Optical Block Detector Assembling.
\end{abstract}

\section{INTRODUCTION}

The general trend in electronic devices development and production is a miniaturization with simultaneous increasing of dissipated heat density. It highlights the importance of an accurate thermal analysis through the design process of electronics. The circuit printed board is a basic element of many electronic devices, and a correct prediction of temperature distribution over its area provides the necessary information for the evaluation of temperature of each component as well as its junction.

In the equipment and instruments for Space application, these boards are often assembled in a package where they are fitted through the perimeter to a structural frame having a good thermal contact. This design conception yields a thermo-mechanical interface to a surface whose temperature is maintained by onboard thermal control sub-system. The frame structure provides a thermal conductive path from dissipated components to this surface acting here as a local heat sink for the each board. External surfaces of the package box are usually covered with multi-layer insulation to block the external radiative flux extremes of Space conditions. During assembling and testing phases as well as before launch, the electronic equipment is submitted to many functional tests. Under such tests, the convective portion of heat transfer contributes to the cooling of electronic boards and, finally, overall temperatures of the components usually are more favorable that under flight condition.

Extracted from this brief depiction, the problem under investigation is the following: to create an analitical tool for prediction of steady-state temperature distribution over a flat plate with multiple spot-type heat dissipations under Dirichlet (1st type) boundary conditions through the perimeter and with additional heat removal by the convection in orthogonal direction.
A straightforward approach is a numerical simulation with a fine grid capable to fit all multiple dissipated components of different dimensions. Finite differences, finite elements or boundary elements techniques can be applied. An alternative approach is the creation of an analytical model of temperature distribution. The analytical approach versus numerical one can give a closed-form expression, which is simple to use in engineering practice and is ready to imbedded in a higherlevel analyzer. In the case of large number of components of small size the numerical approach becomes a hard task because of necessity of drastic refining of the grid. Beside that, the analytical solution can give more valuable information on peculiarities of the board thermal design and provides a physical insight into interaction of different design parameters.

Haque et al, in 1999 used a Fourier series method for obtaining a steady-state temperature mapping over the power electronic building block processor. The method, in spite of being analytical, is realized by the TAMS-A software, developed by Ellison in 1990.

Green's Function technique (Beck, 1992) is very suitable for transient problems, whereas for steady-state problems the integral transform methods seem more adequate. Pesare at al (2001) found an analytical model for 3-D package under several assumptions. A 2-D Fourier transform was applied to simplify the initial equation to the second-order ordinary differential equation. Temperature dependence of thermal conductivity was shifted to the boundary conditions by the Kirchoff transformation and then the 1st order Taylor expansion was applied to the inverse transform. Spatially distributed heat loads from components were approximated as a set of elementary point sources. 
Culham et al in 2000 utilized the Fourier series technique for Laplace statement of heat equation under uniform boundary conditions to obtain a temperature distribution throughout a multi-layer electronic package. The layer-layer and package-board interfaces were considered.

Shukla in 2001 developed the 3D thermal model of a circuit board with discrete surface heat sources by integral transform technique. The solution was compared to the numerical one obtained from the finite element method.

The present work continues the efforts to quest the analytical solutions for the temperature map over plates with multiple heat sources, staying within limits of a $2 \mathrm{D}$ domain. An emphasis is taken upon clarity of utilization, seeking of a simple procedure for evaluation of convection contribution and obtaining an analytical expression for the upper error limit introduced by series truncation. The integral transform technique, developed and generalized by Özisik (1980), Mikhailov (1984) and Cotta (1993), was taken as a baseline for this study rather than Fourier series methods.

\section{ANALYTICAL SOLUTION IN 2D DOMAIN}

The main assumptions made are the following. First, electronic components have a good thermal contact with the base plate. Second, thermal conductivity along the plate area is homogeneous. Third, the heat transfer coefficient for convective heat exchange in orthogonal direction is uniform over the whole area of the plate. Forth, temperatures on boundaries over perimeter of the plate are prescribed and uniform. Fifth, spot heat sources, representing electronic components, have rectangular shape.

Under these assumptions, the heat equation can be expressed in vector form in the following way

$\delta k \nabla^{2} T(\mathbf{r})+q(\mathbf{r})-h_{z}\left(T(\mathbf{r})-T_{\infty}\right)=0 \quad$ in region $\mathrm{R}$

$T(\mathbf{r})=T_{0}$ on boundaries

Where $q(r)$ is the distributed density of heat loads from components $(\mathrm{W} / \mathrm{m} 2)$. When rewriting the equations in rectangular coordinates with respect to a new variable

$$
\Delta T(\mathbf{r})=T(\mathbf{r})-T_{0},
$$

one can obtain an equation with homogeneous boundary conditions (BC) of 1st type

$$
\begin{gathered}
\delta k \frac{\partial^{2} \Delta T}{\partial x^{2}}+\delta k \frac{\partial^{2} \Delta T}{\partial y^{2}}+q(x, y)-h_{z}\left(\Delta T-\Delta T_{\infty}\right)=0 \\
(\mathrm{x}, \mathrm{y}) \in \mathrm{R}
\end{gathered}
$$

$\Delta T=0$ on boundaries.

Assuming the related multidimensional SturmLiouville problem is a separable one in the associated independent variables, $\quad \psi(x, y)=X(x) Y(y)$, the corresponding pair of auxiliary eigenvalue problems can be written as

$$
\frac{\partial^{2} X}{\partial x^{2}}+\beta^{2} X=0 ; \quad \frac{\partial^{2} Y}{\partial y^{2}}+\gamma^{2} Y=0
$$

The integral-transform pair (Ozisik, 1980) is expressed as the following

Integral transform :

$$
\begin{aligned}
\Delta \bar{T}_{m n} & =\Delta \bar{T}\left(\beta_{m}, \gamma_{n}\right)= \\
& \int_{x=0}^{a} \int_{y=0}^{b} X\left(\beta_{m}, x\right) Y\left(\gamma_{n}, y\right) \Delta T(x, y) d x d y
\end{aligned}
$$

Inversion formula :

$$
\Delta T(x, y)=\sum_{m=1}^{\infty} \sum_{n=1}^{\infty} \frac{X\left(\beta_{m}, x\right) Y\left(\gamma_{n}, y\right)}{N\left(\beta_{m}\right) N\left(\gamma_{n}\right)} \Delta \bar{T}\left(\beta_{m}, \gamma_{n}\right)
$$

The normalization integrals are

$$
N\left(\beta_{m}\right)=\int_{x=0}^{a}\left[X\left(\beta_{m}, x\right)\right]^{2} d x ; \quad N\left(\gamma_{n}\right)=\int_{y=0}^{b}\left[Y\left(\gamma_{n}, y\right)\right]^{2} d y
$$

For the homogeneous $\mathrm{BC}$ of 1 st type, all eigen components are known (Ozisik, 1980)

$$
\begin{aligned}
& X\left(\beta_{m}, x\right)=X_{m}=\sin \left(\beta_{m} x\right) ; \quad N\left(\beta_{m}\right)=\frac{a}{2} ; \quad \beta_{m}=\frac{m \pi}{a} ; \\
& Y\left(\gamma_{n}, y\right)=Y_{n}=\sin \left(\gamma_{n} y\right) ; \quad N\left(\gamma_{n}\right)=\frac{b}{2} ; \quad \gamma_{n}=\frac{n \pi}{b} .
\end{aligned}
$$

Where $a$ and $b$ are the dimensions of the plate in $x$ and $y$ directions correspondently; $m$ and $n$ are integer numbers.

Thus, the inversion (Eq. 8) provides the base for a closed analytical expression for the temperature distribution

$$
\Delta T(x, y)=\frac{4}{a b} \sum_{m=1 n=1}^{\infty} \sum_{n=1}^{\infty} \sin \left(m \pi \frac{x}{a}\right) \sin \left(n \pi \frac{y}{b}\right) \Delta \bar{T}\left(\beta_{m}, \gamma_{n}\right)
$$

In spite of the alternative approach, suggested by Mikhailov et al in 1984 for multi-dimension problems, which could give only one infinite sum in this case, the straight approach, that involves double sums, was selected instead due to its symmetry, clarity and simplicity of integrating out the rectangular heat spot sub-domains.

Let multiply the Eq. (3) by $X_{m} Y_{n}$ and integrate over the area of the plate $A$

$$
\begin{aligned}
& \delta k \int_{A} X_{m} Y_{n} \frac{\partial^{2} \Delta T}{\partial x^{2}} d x d y+\delta k \int_{A} X_{m} Y_{n} \frac{\partial^{2} \Delta T}{\partial y^{2}} d x d y+ \\
& \int_{A} X_{m} Y_{n} q(x, y) d x d y- \\
& h_{z} \int_{A} X_{m} Y_{n} \Delta T d x d y+\Delta T_{\infty} h_{z} \int_{A} X_{m} Y_{n} d x d y=0
\end{aligned}
$$

The first two terms can be evaluated by making use of the Green's theorem in its particular case of homogeneous boundary conditions 


$$
\begin{gathered}
\delta k \int_{A} X_{m} Y_{n} \frac{\partial^{2} \Delta T}{\partial x^{2}} d x d y+\delta k \int_{A} X_{m} Y_{n} \frac{\partial^{2} \Delta T}{\partial y^{2}} d x d y= \\
\delta k \int_{A} \Delta T Y_{n} \frac{\partial^{2} X_{m}}{\partial x^{2}} d x d y+\delta k \int_{A} \Delta T X_{m} \frac{\partial^{2} Y_{n}}{\partial y^{2}} d x d y
\end{gathered}
$$

Let multiply the (Eq. 4), expressed for each eigenvalue, by $\Delta T, Y$ then $X$ and integrate over the same region

$$
\begin{aligned}
& \int_{A} \Delta T Y_{n} \frac{\partial^{2} X_{m}}{\partial x^{2}} d x d y+\beta^{2} \int_{A} \Delta T X_{m} Y_{n} d x d y=0 \\
& \int_{A} \Delta T X \frac{\partial^{2} Y_{n}}{\partial y^{2}} d x d y+\gamma^{2} \int_{A} \Delta T X_{m} Y_{n} d x d y=0
\end{aligned}
$$

Combining, one obtains

$$
\begin{aligned}
& \int_{A} X_{m} Y_{n} \frac{\partial^{2} \Delta T}{\partial x^{2}} d x d y+\int_{A} X_{m} Y_{n} \frac{\partial^{2} \Delta T}{\partial y^{2}} d x d y= \\
& \quad-\left(\beta_{m}^{2}+\gamma_{m}^{2}\right) \int_{A} \Delta T X_{m} Y_{n} d x d y=-\left(\beta_{m}^{2}+\gamma_{m}^{2}\right) \Delta \bar{T}_{m n}
\end{aligned}
$$

Now, the original heat equation can be re-written for the transformed temperature difference

$$
\begin{gathered}
-\delta k\left(\beta_{m}^{2}+\gamma_{m}^{2}\right) \Delta \bar{T}_{m n}+\int_{A} X_{m} Y_{n} q(x, y) d x d y-h_{z} \Delta \bar{T}_{m n}+ \\
h_{z} \Delta T_{\infty} \int_{A} X_{m} Y_{n} d x d y=0
\end{gathered}
$$

It is a simple algebraic linear equation with regard to the transformed variable.

Now let perform the integration of the rest source terms. The integral of eigenfunction is

$$
\begin{aligned}
& \int_{A} X_{m} Y_{n} d x d y=\int_{x=0}^{a} \int_{y=0}^{b} \sin \left(m \pi \frac{x}{a}\right) \sin \left(n \pi \frac{y}{b}\right) d y d x= \\
& a b \frac{(1-\cos (m \pi))(1-\cos (n \pi))}{m n \pi^{2}}=\frac{a b}{\pi^{2}} \omega_{m n} \\
& \text { where } \omega_{m n}=\frac{1-(-1)^{m}-(-1)^{n}+(-1)^{m+n}}{m n}
\end{aligned}
$$

Density distribution function is assumed to be discrete and homogeneous over its rectangular region. For each $\mathrm{j}$-th heat load with coordinates $\left(\xi_{1 \mathrm{j}}, \zeta_{1 \mathrm{j}}\right)$ for its left bottom corner and $\left(\xi_{2 \mathrm{j}}, \zeta_{2 \mathrm{j}}\right)$ for its right upper corner, the heat flux density is

$$
q_{j}(x, y)=\left\{\begin{array}{l}
q_{j} \text { for } x \in\left[\xi_{1 j}, \xi_{2 j}\right] \cap y \in\left[\zeta_{1 j}, \zeta_{2 j}\right] \\
0 \text { otherwise }
\end{array}\right.
$$

and

$$
q(x, y)=\sum_{j=1}^{J} q_{j}(x, y)
$$

The complete integral over all $\mathrm{J}$ heat sources is

$$
\begin{aligned}
& \int_{A} X_{m} Y_{n} q(x, y) d x d y= \\
& \sum_{j=1}^{J} q_{j} \int_{x=\xi_{1 j}}^{\xi_{2 j}} \int_{y=\varsigma_{1 j}}^{\varsigma_{2 j}} \sin \left(m \pi \frac{x}{a}\right) \sin \left(n \pi \frac{y}{b}\right) d y d x=\sum_{j=1}^{J} \frac{a b}{\pi^{2}} q_{j} \Omega_{m n j} \\
& \text { where } \Omega_{m n j}=\frac{1}{m n}\left[\cos \left(m \pi \frac{\xi_{1 j}}{a}\right)-\cos \left(m \pi \frac{\xi_{2 j}}{a}\right)\right] \\
& {\left[\cos \left(n \pi \frac{\zeta_{1 j}}{b}\right)-\cos \left(m \pi \frac{\zeta_{2 j}}{b}\right)\right]}
\end{aligned}
$$

Now, the integral transform for temperature difference can be written as

$$
\begin{aligned}
& \Delta \bar{T}\left(\beta_{m}, \gamma_{n}\right)= \\
& \frac{a^{3} b^{3}}{\pi^{2}\left(k \delta \pi^{2}\left(m^{2} b^{2}+n^{2} a^{2}\right)+a^{2} b^{2} h_{z}\right)} . \\
& {\left[h_{z} \Delta T_{z} \omega_{n m}+\sum_{j=1}^{J} q_{j} \Omega_{j m n}\right]}
\end{aligned}
$$

Finally, the 2-D solution is the following

$$
\begin{gathered}
\Delta T(x, y)= \\
\frac{4 a^{2} b^{2}}{\pi^{2}} \sum_{m=1}^{\infty} \sum_{n=1}^{\infty} \frac{\sin \left(m \pi \frac{x}{a}\right) \sin \left(n \pi \frac{y}{b}\right)}{\left[k \delta \pi^{2}\left(m^{2} b^{2}+n^{2} a^{2}\right)+a^{2} b^{2} h_{z}\right]} . \\
\left(h_{z} \Delta T_{z} \omega_{n m}+\sum_{j=1}^{J} q_{j} \Omega_{j m n}\right)
\end{gathered}
$$

This solution yields the temperature map over a plate as a closed analytical function of all design parameters.

\section{CONVECTION AND CONDUCTION CONTRIBUTIONS}

The contribution of convection and conduction portions of heat transfer can be acquired from the obtained solution for each electronic component as well as for the entire plate by direct integration. The convection portion can be obtained by integration over the surface of interest. The conduction portion can be defined through integration of thermal gradients along a closed contour. These calculations provide also an additional mean for estimation of truncation errors in term of heat flux values through verification of overall balance.

By selecting this contour fitted to the bounds of $j$ th heat source, the conductive portion of heat flux can be defined as a sum of fluxes for each of the four sides:

$$
\begin{aligned}
& Q_{k j}=k \delta\left(\left.\int_{\zeta_{1 j}}^{\zeta_{2 j}} \frac{\partial \Delta T}{\partial x}\right|_{x=\xi_{1 j}} d y-\right. \\
& \left.\left.\int_{\zeta_{1 j}}^{\zeta_{2 j}} \frac{\partial \Delta T}{\partial x}\right|_{x=\xi_{2 j}}+\left.\int_{\xi_{1 j}}^{\xi_{2 j}} \frac{\partial \Delta T}{\partial y}\right|_{y=\zeta_{1 j}} d x-\left.\int_{\xi_{1 j}}^{\xi_{2 j}} \frac{\partial \Delta T}{\partial y}\right|_{y=\zeta_{2 j}} d x\right)
\end{aligned}
$$


CIÊNCIA/SCIENCE

The convection portion is obtained by integration over the appropriate surface:

$Q_{h j}=h_{z} \int_{\xi_{1 j}}^{\xi_{2 j}} \int_{\zeta_{1 j}}^{\zeta_{2 j}} \Delta T d y d x-h_{z} A_{j}\left(T_{\infty}-T_{0}\right)$

The relative error in terms of heat flux for each electronic component is defined as

$$
\delta \bar{Q}_{j}=\left|1-\frac{Q_{k j}}{Q_{j}}-\frac{Q_{h j}}{Q_{j}}\right|
$$

The solution re-written in the compact form is:

$$
\begin{aligned}
& \Delta T(x, y)=\frac{4}{\pi^{2}} \sum_{m=1}^{\infty} \sum_{n=1}^{\infty} \Phi_{m n} \sin \left(m \pi \frac{x}{a}\right) \sin \left(n \pi \frac{y}{b}\right) \\
& \Phi_{\mathrm{mn}}=\frac{a^{2} b^{2}}{\left[k \delta \pi^{2}\left(m^{2} b^{2}+n^{2} a^{2}\right)+a^{2} b^{2} h_{z}\right]} \cdot \\
& \left(h_{z} \Delta T_{z} \omega_{n m}+\sum_{j=1}^{J} q_{j} \Omega_{j m n}\right)
\end{aligned}
$$

Using this function and performing differentiation-integration by (Eq. 20), one can obtain the conduction contribution for $\mathrm{j}$-th source

$$
\begin{aligned}
Q_{k j}= & \frac{4 k \delta}{\pi^{2}} \sum_{m=1}^{\infty} \sum_{n=1}^{\infty} \Phi_{m n} \frac{\left(a^{2} n^{2}+b^{2} m^{2}\right)}{a b m n} . \\
& {\left[\cos \left(m \pi \frac{\xi_{1 j}}{a}\right)-\cos \left(m \pi \frac{\xi_{2 j}}{a}\right)\right] . } \\
& {\left[\cos \left(n \pi \frac{\zeta_{1 j}}{b}\right)-\cos \left(m \pi \frac{\zeta_{2 j}}{b}\right)\right] }
\end{aligned}
$$

Or, using the previously defined function $\Omega$, Eq.(17), the compact form of this expression is

$$
Q_{k j}=\frac{4 k \delta}{a b \pi^{2}} \sum_{m=1}^{\infty} \sum_{n=1}^{\infty}\left(a^{2} n^{2}+b^{2} m^{2}\right) \Phi_{m n} \Omega_{m n j}
$$

The overall balance of conduction portion over the whole plate has a similar-form result equation

$$
Q_{k}=\frac{4 k \delta}{a b \pi^{2}} \sum_{m=1}^{\infty} \sum_{n=1}^{\infty}\left(a^{2} n^{2}+b^{2} m^{2}\right) \Phi_{m n} \omega_{m n}
$$

The corresponding convection contributions are the following:

$$
\begin{gathered}
Q_{h j}=\frac{4 a b h_{z}}{\pi^{4}} \sum_{m=1}^{\infty} \sum_{n=1}^{\infty} \Phi_{m n} \Omega_{m n j}-h_{z} A_{j}\left(T_{\infty}-T_{0}\right) \\
Q_{h}=\frac{4 a b h_{z}}{\pi^{4}} \sum_{m=1}^{\infty} \sum_{n=1}^{\infty} \Phi_{m n} \omega_{m n}-h_{z} A\left(T_{\infty}-T_{0}\right)
\end{gathered}
$$

The relative error in terms of heat flux for the whole plate is defined as

$$
\delta \bar{Q}=\left|1-\frac{Q_{k}+Q_{h}}{\sum_{j=1}^{J} Q_{j}}\right|
$$

The last equation gives exact magnitude of the overall heat dis-balance introduced by truncation of series for a given set of input parameters.

\section{ERROR SUPREMUM}

Now we will estimate the residue of the series to obtain (conservative) upper limit of the error due to series truncation as an analytical function. Expression for the error of truncation is the following

$$
\begin{gathered}
\delta^{*}(\Delta T(x, y))=\frac{4}{\pi^{2}} \sum_{m=M n=N}^{\infty} \sum_{m n}^{\infty} \widetilde{\Phi}_{m n} \\
\widetilde{\Phi}_{m n}=\Phi_{m n} \sin \left(m \pi \frac{x}{a}\right) \sin \left(n \pi \frac{y}{b}\right)
\end{gathered}
$$

Where $(M-1)$ and $(N-1)$ are number of terms in corresponding finite series.

For relatively large $M$ and $N$ the estimation of the supreme of $F$ can be accomplished by setting terms of nominator to their maximum and the terms of denominator to their minimum

$$
\sup \left(\omega_{m n}\right)=\frac{2}{m n} ; \quad \sup \left(\Omega_{m n j}\right)=\frac{4}{m n} ;
$$

Taking into account that $\max (\operatorname{Sin}())=$.1 , it is possible to obtain

$$
\sup \left(\widetilde{\Phi}_{m n}\right)=\frac{\left(2 h_{z} \Delta T_{z}+4 \sum_{j=1}^{J} q_{j}\right)}{k \delta \pi^{2} n m\left(\frac{m^{2}}{a^{2}}+\frac{n^{2}}{b^{2}}\right)}
$$

Now the double sum can be evaluated by

$$
\begin{gathered}
\sum_{m=M}^{\infty} \sum_{n=N}^{\infty} \widetilde{\Phi}_{m n}<\sum_{m=M}^{\infty} \sum_{n=N}^{\infty} \sup \left(\widetilde{\Phi}_{m n}\right)= \\
\frac{a^{2}}{k \delta \pi^{2}}\left(2 h_{z} \Delta T_{z}+4 \sum_{j=1}^{J} q_{j}\right) \Psi
\end{gathered}
$$

where $\Psi=\sum_{m=M}^{\infty} \sum_{n=N}^{\infty} \frac{1}{n m\left(m^{2}+r^{2} n^{2}\right)}$

where $a$ is a largest board side size and $\mathrm{r}=\mathrm{a} / \mathrm{b}$.

As soon as $r \geq 1$, a conservative estimation can be obtained, using the geometric inequality

$$
\frac{1}{n m\left(m^{2}+r^{2} n^{2}\right)}<\frac{1}{n m\left(m^{2}+n^{2}\right)} \leq \frac{1}{2 m^{2} n^{2}}
$$




\section{CIÊNCIA/SCIENCE}

Now the sum of double infinite series can be expressed via Euler Gamma functions

$$
\begin{aligned}
& \Psi \leq \sum_{m=M}^{\infty} \sum_{n=N}^{\infty} \frac{1}{m^{2} n^{2}}=\gamma(M, N) \\
& \gamma(M, N)=\frac{\left(\Gamma^{\prime}(M)^{2}-\Gamma(M) \Gamma^{\prime \prime}(M)\right)\left(\Gamma^{\prime}(N)^{2}-\Gamma(N) \Gamma^{\prime \prime}(N)\right)}{\Gamma(M)^{2} \Gamma(N)^{2}} \\
& \Gamma(z)=\int_{0}^{\infty} t^{z-1} e^{-t} d t ; \quad \Gamma^{\prime}(z)=(z-1) \int_{0}^{\infty} t^{z-2} e^{-t} d t \\
& \Gamma^{\prime \prime}(z)=\left(z^{2}-3 z+2\right) \int_{0}^{\infty} t^{z-3} e^{-t} d t
\end{aligned}
$$

The presented Euler Gamma functions are incorporated in the Mathematica package, Wolfram, 1991.

The final analytical expression for the truncation error is the following

$\sup \delta^{*}=\frac{2 a^{2}}{k \delta \pi^{4}}\left(2 h_{z} \Delta T_{z}+4 \sum_{j=1}^{J} q_{j}\right) \gamma(M, N)$

This is a very important result, which clearly shows how design parameters may make influence to the introducing error because of series truncation.

Some values of the function $\gamma$ are presented in the table.

Table 1. Values of the function $\gamma$

\begin{tabular}{|l|c|c|c|c|c|c|c|}
\hline $\mathrm{M}$ & 5 & 10 & $20(10)$ & 20 & 30 & 50 & 100 \\
\hline $\mathrm{N}$ & 5 & 10 & $10(20)$ & 20 & 30 & 50 & 100 \\
\hline$\gamma$ & $2 \mathrm{E}-2$ & $6 \mathrm{E}-3$ & $3 \mathrm{E}-3$ & $1 \mathrm{E}-3$ & $6 \mathrm{E}-4$ & $2 \mathrm{e}-4$ & $5 \mathrm{E}-5$ \\
\hline
\end{tabular}

For typical values $\mathrm{k}=5 \mathrm{~W} / \mathrm{m} / \mathrm{K}, \delta=0.001 \mathrm{~m}, \mathrm{~h}_{\mathrm{z}}=5$ $\mathrm{W} / \mathrm{m}^{2} / \mathrm{K}, \Delta \mathrm{T}_{\mathrm{z}}=10 \mathrm{~K}, \mathrm{~J}=10, \operatorname{Sum}\left(\mathrm{q}_{\mathrm{j}}\right)=20000 \mathrm{~W} / \mathrm{m} 2, \mathrm{a}=0.2 \mathrm{~m}$; the error evaluation is $\sup \delta^{*} \approx 13800_{*}$. The last, for instant, means that for $\mathrm{N}=\mathrm{M}=100$ we have $\delta^{*}<0.7 \mathrm{~K}$.

Note, it is a very conservative estimation. If we suppose for example, the electronic components cover less than $25 \%$ of whole electronic board area (i.e. $\sup \left(\Omega_{m n}\right)=1$ instead 4$)$, the estimation of error for this case will be $\delta^{*}<0.18 \mathrm{~K}$.

\section{RESULTS}

The model was used for temperature mapping of the electronic board of the DC/DC converter of the CIMEX experiment CEP block.

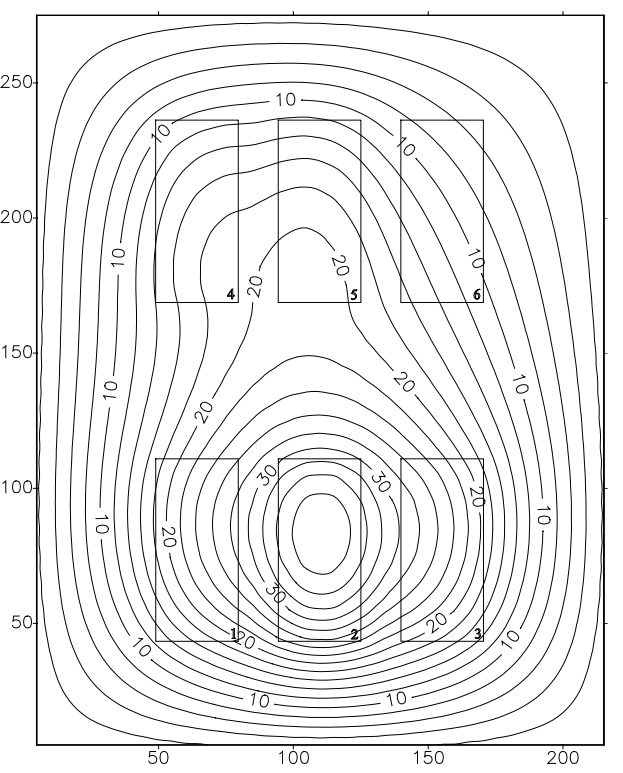

Figure 1. Maximal dissipation without convection cooling

The board has 6 dissipated elements with good thermal contact to base surface. Figure 1 shows contour lines for vacuum conditions at high case dissipation. Maximal temperature differences above base frame temperature $\mathrm{T}_{0}$ was obtained $37.7{ }^{0} \mathrm{C}$.

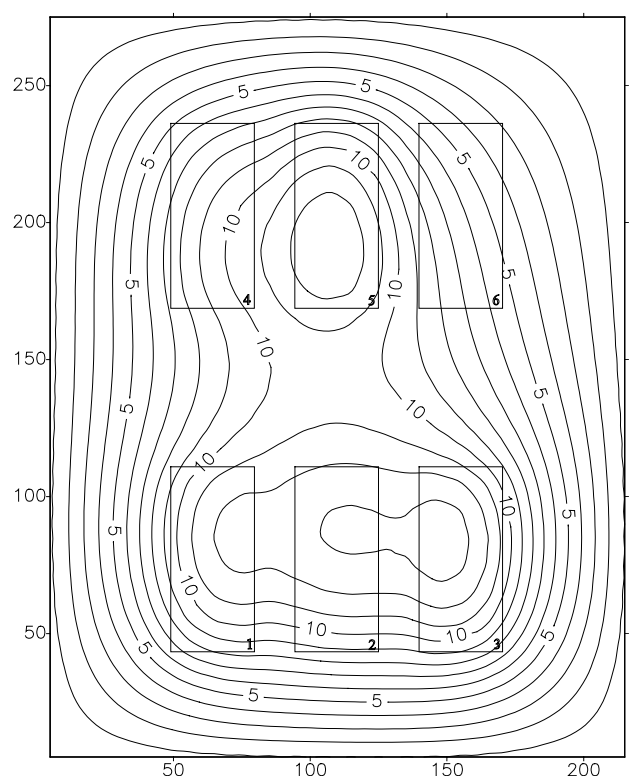

Figure 2. Combined conduction and convection cooling, minimal dissipation

Figure 2 shows contours for the case of low heat dissipation under combined conduction and convection cooling.

The Figure 3 shows a 3D view of the last case. Contour lines are plotted in a $2 \mathrm{~K}$ interval. The dissipations and coordinates of electronic components are presented in the Table 2. 


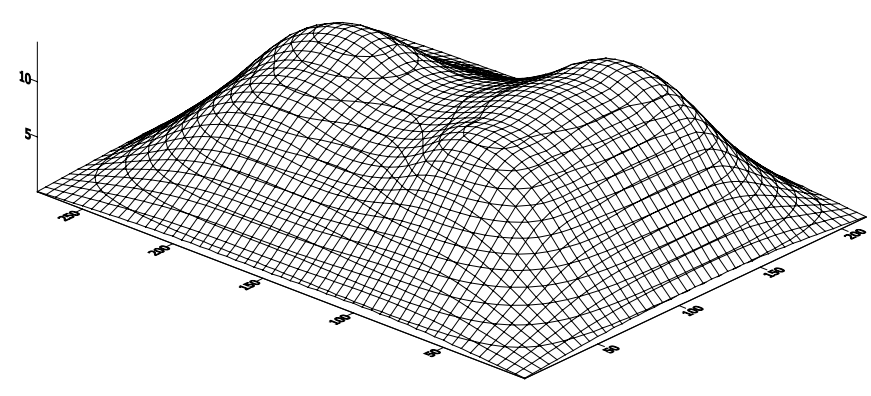

Figure 3. 3D view for the temperature surface of the board for the case of minimal dissipations.

Table 2. Input data for the dissipation components of the DC/DC board

\begin{tabular}{|c|c|c|c|c|c|c|}
\hline $\mathrm{n} / \mathrm{n}$ & $\mathrm{x} 1[\mathrm{~mm}]$ & $\mathrm{x} 2[\mathrm{~mm}]$ & $\mathrm{y} 1[\mathrm{~mm}]$ & $\mathrm{y} 2[\mathrm{~mm}]$ & Qmin [W] & Qmax[W] \\
\hline 1 & 46.5 & 78.4 & 40 & 110.1 & 2.8 & 2.8 \\
\hline 2 & 94 & 126 & 40 & 110.1 & 1.5 & 8.9 \\
\hline 3 & 141.5 & 173.5 & 40 & 110.1 & 3.5 & 3.5 \\
\hline 4 & 46.5 & 78.4 & 169.9 & 240 & 1.5 & 2.8 \\
\hline 5 & 94 & 126 & 169.9 & 240 & 3.1 & 3.1 \\
\hline 6 & 141.5 & 173.5 & 169.9 & 240 & 0 & 0 \\
\hline
\end{tabular}

The general input data, used in all of these calculations, were the following: the board size is $220 \times 280$ $\mathrm{mm}, \mathrm{k}=60 \mathrm{~W} / \mathrm{m} / \mathrm{K}, \delta=0.0016 \mathrm{~m}, \mathrm{~h}_{\mathrm{z}}=0$ or $5 \mathrm{~W} / \mathrm{m}^{2} / \mathrm{K}, \Delta \mathrm{T}_{\mathrm{z}}=0 \mathrm{C}$

\section{CONCLUSIONS}

The developed analytical model provides a relatively simple and clear tool for 2D temperature mapping of circuit boards with electronic components having a good thermal contact to the base plate. Convection cooling is also taken into account. As soon as the exact analytical expression for the upper limit of truncation error is available, the obtained solution can be used as a benchmark for the evaluation of numeric solution of such problems.

A separate evaluation of the contribution of different mechanisms of heat transfer to the overall heat balance gives the designer an important information about how to evaluate the efficiency of adopted means of thermal control.

\section{REFERENCES}

Beck, J.V., Cole, K.D., Haji-Sheikh, A., Litkouhi, B., 1992, "Heat conduction using Green's functions", Hemisphere publishing.

Cotta, R.M., 1993, "Integral transforms in Computational Heat and Fluid Flow",CRC Press, USA.

Culham, J.R., Yovanovich, M.M., Lemczyk, T.F., 2000, "Thermal Characterization of Electronic Packaging Using a Three-Dimensional Fourier Series Solution", Journal of Electronic Packaging, Vol.122, pp. 233-239.

Ellison G.N., 1990, “TAMS-A Thermal analyzer for multilayered structures", Electrosoft, Vol.1, pp. 85-97.

Haque, S., Stinnet, W.A., Nelson, D.J., Lu, G-Q., 1999, "Thermal management of power electronics modules packaged by a stacked-plate technique", Microelectronics Reliability, Vol.39, pp. 1343-1349

Mikhailov, M.D., Ozisik, M.N., 1984, “An Alternative General Solution of the Steady-State Heat Diffusion Equation", John Willey, NY. NY.

Ozisik, M.N., 1980, "Heat Conduction”, John Wiley,

Pesare, M., Giorgio, A., Perri, A.G., 2001, “An analytical method for the thermal laypout optimization of multilayer structure solid-state devices", Solid-State Electronics, Vol.45, pp. 511-517.

Shukla, K.N., 2001, "Thermal Math Modeling and Analysis of an Electronic Assembly", Journal of Electronic Packaging, Vol.123, pp. 372-378.

Wolfram, S., 1991, "Mathematica”, Addison Wesley. 